\title{
Nerve Fibers in the Tumor Microenvironment as a Novel Biomarker for Oncological Outcome in Patients Undergoing Surgery for Perihilar Cholangiocarcinoma
}

\author{
Jan Bednarsch ${ }^{a}$ Jakob Kather ${ }^{b}$ Xiuxiang Tan ${ }^{a, c, d}$ Shivan Sivakumar ${ }^{e, f}$ \\ Claudio Cacchi ${ }^{9}$ Georg Wiltberger ${ }^{a}$ Zoltan Czigany ${ }^{a}$ Florian Ulmer $^{a}$ \\ Ulf Peter Neumann ${ }^{a, c}$ Lara Rosaline Heija, d, g
}

aDepartment of Surgery and Transplantation, University Hospital RWTH Aachen, Aachen, Germany; ${ }^{b}$ Division of Gastroenterology and Hepatology, Department of Internal Medicine III, University Hospital RWTH Aachen, Aachen, Germany; 'Department of Surgery, Maastricht University Medical Center (MUMC), Maastricht, The Netherlands; ${ }^{\mathrm{d}}$ NUTRIM School of Nutrition and Translational Research in Metabolism, Maastricht University, Maastricht, The Netherlands; ' Department of Oncology, University of Oxford, Oxford, UK; ' Kennedy Institute of Rheumatology, University of Oxford, Oxford, UK; IInstitute of Pathology, University Hospital RWTH Aachen, Aachen, Germany

\author{
Keywords \\ Cholangiocarcinoma $\cdot$ Nerve fiber density $\cdot$ Oncological \\ outcome $\cdot$ Biomarker
}

\begin{abstract}
Introduction: Perihilar cholangiocarcinoma (pCCA) is a biliary tract cancer with a dismal prognosis, with surgery being the only chance of cure. A characteristic aggressive biological feature of pCCA is perineural growth which is defined by the invasion of cancer cells to nerves and nerve fibers. Recently, nerve fiber density (NFD) was linked to oncological outcomes in various malignancies; however, its prognostic role in pCCA remains to be elucidated. Materials and Methods: Data of 101 pCCA patients who underwent curativeintent surgery between 2010 and 2019 were included in this study. Extensive group comparisons between patients with high and low NFD were carried out, and the association of cancer-specific survival (CSS) and recurrence-free survival with NFD and other clinicopathological characteristics was assessed using univariate and multivariable cox regression
\end{abstract}

models. Results: Patients with high NFD showed a median CSS of 90 months (95\% Cl: 48-132, 3-year CSS = 77\%, 5-year CSS $=72 \%$ ) compared to 33 months (95\% Cl: 19-47, 3-year CSS $=46 \%, 5$-year CSS $=32 \%)$ in patients with low NFD ( $p=$ 0.006 log rank). Further, N1 category (HR $=2.84, p=0.001)$ and high NFD ( $H R=0.41, p=0.024)$ were identified as independent predictors of CSS in multivariable analysis. Patients with high NFD and negative lymph nodes showed a median CSS of 90 months (3-year CSS $=88 \%$, 5-year CSS $=80 \%$ ), while patients with either positive lymph nodes or low NFD displayed a median CSS of 51 months (3-year CSS $=59 \%$, 5 -year CSS $=45 \%$ ) and patients with both positive lymph nodes and low NFD a median CSS of 24 months (3-year CSS $=26 \%$, 5-year CSS $=16 \%, p=0.001$ log rank). Conclusion: NFD has been identified as an important novel prognostic biomarker in pCCA patients. NFD alone and in combination with nodal status in particular allows to stratify pCCA patients based on their risk for inferior oncological outcomes after curative-intent surgery.

(C) 2021 The Author(s) Published by S. Karger AG, Basel karger@karger.com www.karger.com/lic

Karger $\stackrel{\text { ' }}{5}$

GOPEN ACCESS
(C) 2021 The Author(s)

Published by S. Karger AG, Basel

This is an Open Access article licensed under the Creative Commons Attribution-NonCommercial-4.0 International License (CC BY-NC) (http://www.karger.com/Services/OpenAccessLicense), applicable to the online version of the article only. Usage and distribution for commercial purposes requires written permission.
Correspondence to:

Lara Rosaline Heij, lheij@ukaachen.de 


\section{Introduction}

Perihilar cholangiocarcinoma (pCCA) is the commonest subtype among biliary tract tumors and is associated with a poor prognosis [1-3]. Liver resection with vascular reconstructions and radical lymphadenectomy emerged as the gold standard of therapy in resectable disease, and this yielded improved survival rates in selected cohorts [3-9]. Despite these encouraging outcomes, surgical therapy remains challenging and often displays significant perioperative mortality rates exceeding $10 \%$ due to the distinct anatomic location of the tumor and the close proximity to major vascular structures [8-11].

As the prognosis spans from poor prognosis to good prognosis, there is significant value in being able to identify prognostic features [3]. Tumor differentiation, R0 status, and lymph node status have been previously reported as important prognostic factors by Nagino et al. [12]. Our group and others have confirmed perioperative blood transfusion, serum albumin, and lymphovascular invasion (LVI) as independent variables predicting adverse outcomes following surgery for pCCA $[3,13,14]$.

PCCA is characterized by a large desmoplastic stroma component which might explain systemic therapy resistance [15]. Nerve fibers are a component of the tumor microenvironment (TME), and a significant proportion of cholangiocarcinoma patients display a tumor infiltration of the epineural, perineural, and endoneural space of the neural sheath $[16,17]$. These features are termed perineural infiltration (PNI). PNI can be recognized on Hematoxylin and Eosin $(\mathrm{H} \& \mathrm{E})$ staining and appears to be an independent predictor of prognosis in cholangiocarcinoma (CCA). In contrast, the prognostic role of nerve fiber density (NFD) referring to small nerve fibers in the TME which are usually not visible on H\&E staining and do not show invasion of cancer cells remains to be determined in pCCA patients $[18,19]$. Therefore, we aimed to investigate NFD as a prognostic marker in a large European cohort of pCCA patients undergoing surgical resection.

\section{Materials and Methods}

\section{Patients}

All consecutive patients with pCCA who underwent surgical resection at the University Hospital RWTH Aachen (UH-RWTH) between 2010 and 2019 were eligible for this study. Of these patients $(n=127), 20$ individuals were excluded $(n=14$ perioperative mortality; $n=6$ with missing NFD data). Subsequently, a cohort of 101 patients was included in this analysis (Fig. 1b). The study was conducted in accordance with the requirements of the Institution- al Review Board of the RWTH-Aachen University (EK 106/18), the Declaration of Helsinki, and good clinical practice guidelines (ICH-GCP).

\section{Staging and Surgical Technique}

All patients who were referred for surgical treatment for pCCA to our institution underwent a detailed clinical workup as previously described $[9,20]$. In brief, tumor anatomy and localization were assessed by endoscopic retrograde cholangiopancreatography or magnetic resonance cholangiopancreatography, while the presence of distant metastases was ruled out by multiphase CT. Additionally, the vascular anatomy and the potential invasion of major vessels at the liver hilum were also assessed by multiphase CT.

Our preoperative workup included a unilateral stenting strategy as the standard of care to relieve the future liver remnant from cholestasis and bilateral stenting in cases with persisting cholangitis. Endoscopic biliary drainage was generally preferred over percutaneous biliary drainage. In patients with insufficient future liver remnant scheduled for right-sided hepatectomy, a right portal vein embolization was conducted 2-4 weeks before surgery. The decision for surgery as primary treatment for the cancer was made by an experienced hepatobiliary surgeon and approved by the local multidisciplinary tumor board in all cases.

The surgical procedure was carried out as previously described by Neuhaus et al. [3, 8, 9, 21, 22]. In briefly, a "no-touch" hilar en bloc resection approach, as defined by liver resection with mandatory portal vein resection and reconstruction, was carried out in all cases. Additional arterial resection and reconstruction as well as the concomitant resection of the pancreatic head (hepatoduodenopancreatectomy) was necessary in selected cases (Table 1). Lymphadenectomy comprising the pericholedochal, the periportal, the common hepatic lymph nodes, the posterior pancreaticoduodenal, and the celiac lymph nodes was routinely performed. All surgical specimens were evaluated by an experienced staff pathologist.

Follow-Up

Adjuvant therapy was recommended for patients displaying high risk features (e.g., positive nodal status or R1 resection) from 2010 to 2017 and later in every individual in accordance with the results of the BILCAP trial [23]. Each patient underwent a regular follow-up including clinical examinations, standard laboratory blood tests with tumor markers (CA 19-9), and cross-sectional imaging by the referring oncologist or the local outpatient clinic. If tumor recurrence was suspected, additional imaging and/or biopsy was performed to confirm the diagnosis.

\section{Assessment of NFD}

Immunohistochemistry was performed on formalin-fixed, paraffin-embedded tissue sections as previously described [19]. In brief, sections ( $2.5 \mu \mathrm{m}$ thick) were cut, deparaffinized in xylene, and rehydrated in graded alcohols. Slides were boiled in citrate buffer ( $\mathrm{pH} 6.0$ ) at $95-100^{\circ} \mathrm{C}$ for $5 \mathrm{~min}$ and were cooled for $20 \mathrm{~min}$ and with endogenous peroxide in methanol for $10 \mathrm{~min}$. Sections were incubated with rabbit anti-human PGP 9.5 (DAKO 1:100) overnight at $4^{\circ} \mathrm{C}$. A single digital image was uploaded in Qupath 0.1.6. As previously described, all slides were assessed by a trained pathologist who was blinded to the clinical outcomes of the individual patients, and NFD was evaluated by manually counting the number of nerve fascicles with diameters of $<100 \mu \mathrm{m}$ in 20 continuous visual fields at $\times 200$ magnification [19]. An overview of 


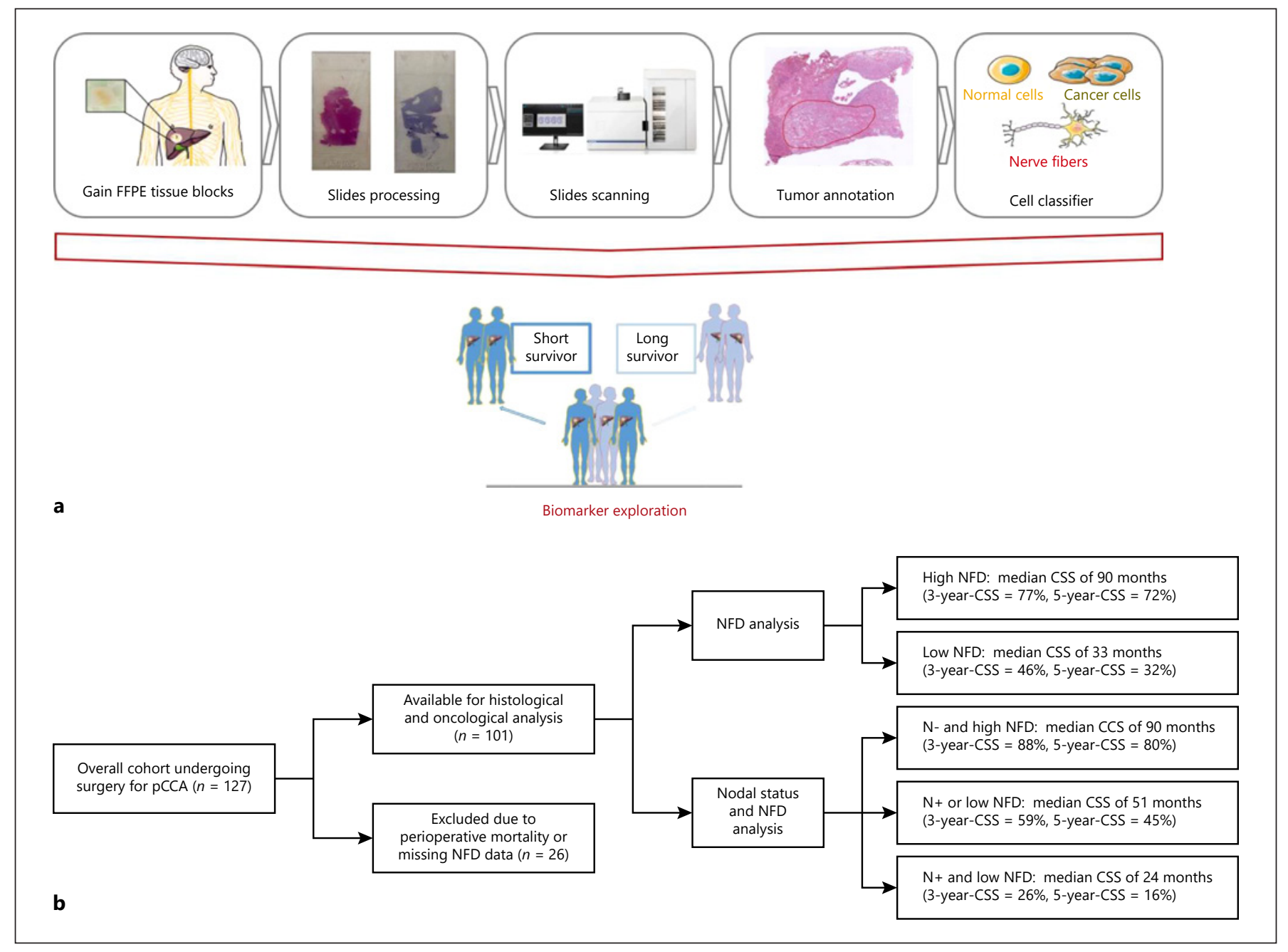

Fig. 1. Workflow and study cohort. a Overview of all steps from tissue to biomarker. FFPE, formalin-fixed paraffin-embedded. b Study cohort. CSS, cancer-specific survival; NFD, nerve fiber density; pCCA, perihilar cholangiocarcinoma.

the workflow is presented in Figure 1a. Based on NFD results, patients were categorized into a low NFD group ( $<10$ nerve fibers) and a high NFD group ( $\geq 10$ nerve fibers).

\section{Assessment of Origin of the Small Nerve Fibers}

Immunohistochemistry was performed on formalin-fixed, paraffin-embedded tissue sections as previously described. Sections were incubated with neuropeptide Y (Abcam 1:200) and vasoactive intestinal peptide (Abcam 1:50) overnight at $4^{\circ} \mathrm{C}$.

\section{Statistical Analysis}

The primary endpoint of this study was cancer-specific survival (CSS), which was defined from the date of resection to the date of tumor-specific death. Deaths not associated with the tumor, for example, cardiovascular events, were censored at the time of death. The secondary endpoint was recurrence-free survival (RFS), which was defined as the period from surgery to the date of first recur- rence. Patients without tumor recurrence were censored at the time of death or at the last follow-up. Perioperative mortality was defined as in-hospital mortality. The cutoff level for NFD categorization was determined by the receiver operating characteristic analysis of CCS with respect to NFD as previously described [19]. Group comparisons were conducted by the Mann-Whitney U test in case of continuous variables, while the $\chi^{2}$ test, Fisher's exact test, or linear-by-linear association in accordance with scale and number count were used in case of categorical variables. The associations of CSS and RFS with clinicopathological characteristics were assessed using univariate and multivariable Cox regression analyses in a backward selection model. Survival curves were generated by the Kaplan-Meier method and compared with the log-rank test. Median follow-up was assessed with the reverse Kaplan-Meier method. The level of significance was set to $p<0.05$, and $p$ values were given for 2 -sided testing. Analyses were performed using SPSS Statistics 24 (IBM Corp., Armonk, NY, USA). 
Table 1. Patients' characteristics

\begin{tabular}{|c|c|c|c|c|}
\hline Demographics & $\begin{array}{l}\text { Overall cohort } \\
(n=101)\end{array}$ & $\begin{array}{l}\text { High NFD } \\
(n=31)\end{array}$ & $\begin{array}{l}\text { Low NFD } \\
(n=70)\end{array}$ & $p$ value \\
\hline Gender, male/female (\%) & $68(67) / 33(33)$ & $22(71) / 9(29)$ & $46(66) / 24(34)$ & 0.604 \\
\hline Age, years & $68(57-74)$ & $68(56-72)$ & $68(57-74)$ & 0.968 \\
\hline $\mathrm{BMI}, \mathrm{kg} / \mathrm{m}^{2}$ & $25(22-29)$ & $25(23-30)$ & $25(22-28)$ & 0.439 \\
\hline Neoadjuvant therapy, $n(\%)$ & $4(4)$ & $3(10)$ & $1(1)$ & 0.050 \\
\hline PVE, $n(\%)$ & $43(43)$ & $12(39)$ & $31(44)$ & 0.601 \\
\hline I & $4(4)$ & $1(3)$ & $3(4)$ & \multirow{5}{*}{0.740} \\
\hline II & $44(44)$ & $16(52)$ & $28(40)$ & \\
\hline III & $50(50)$ & $13(42)$ & $37(53)$ & \\
\hline IV & $3(3)$ & $1(3)$ & $2(3)$ & \\
\hline $\mathrm{V}$ & 0 & 0 & & \\
\hline \multicolumn{5}{|l|}{ Bismuth classification, $n(\%)$} \\
\hline IV & $32(32)$ & $10(32)$ & $22(31)$ & 0.675 \\
\hline Preoperative cholangitis, $n(\%)$ & $27(27)$ & $5(16)$ & $22(31)$ & 0.109 \\
\hline Preoperative PBD, $n(\%)$ & $23(23)$ & $5(16)$ & $18(26)$ & 0.289 \\
\hline Preoperative EBD, $n(\%)$ & $78(77)$ & $23(74)$ & $55(79)$ & 0.628 \\
\hline \multicolumn{5}{|l|}{ Clinical chemistry } \\
\hline Albumin, g/dL & $37(33-42)$ & $39(34-42)$ & $37(32-41)$ & 0.268 \\
\hline AST, U/L & $47(36-81)$ & $49(32-135)$ & $47(36-77)$ & 0.932 \\
\hline $\mathrm{ALT}, \mathrm{U} / \mathrm{L}$ & $68(37-133)$ & $88(43-161)$ & $63(37-120)$ & 0.318 \\
\hline GGT, U/L & $451(221-774)$ & $554(240-982)$ & $441(209-756)$ & 0.304 \\
\hline Total bilirubin, mg/dL & $1.2(0.6-2.8)$ & $1.2(0.8-2.6)$ & $1.2(0.5-2.8)$ & 0.805 \\
\hline Platelet count, $/ \mathrm{nL}$ & $296(228-393)$ & $292(212-392)$ & $297(230-399)$ & 0.548 \\
\hline Alkaline phosphatase, U/L & $254(169-396)$ & $251(160-445)$ & $254(183-372)$ & 0.747 \\
\hline Limited bile duct resection & $1(1)$ & 0 & $1(1)$ & \multirow{9}{*}{0.475} \\
\hline Right hepatectomy & $11(11)$ & $2(7)$ & $9(13)$ & \\
\hline Left hepatectomy & $11(11)$ & $5(16)$ & $6(9)$ & \\
\hline Extended right hepatectomy & $18(18)$ & $6(19)$ & $12(17)$ & \\
\hline Extended left hepatectomy & $28(28)$ & $9(29)$ & $19(27)$ & \\
\hline Right trisectionectomy & $20(20)$ & $6(19)$ & $14(20)$ & \\
\hline Left trisectionectomy & $5(5)$ & $3(10)$ & $2(3)$ & \\
\hline Hepatoduodenoectomy & $6(6)$ & 0 & $6(9)$ & \\
\hline ALPPS & $1(1)$ & 0 & $1(1)$ & \\
\hline Portal vein reconstruction & $101(100)$ & $31(100)$ & $70(100)$ & 0.999 \\
\hline Arterial reconstruction & $7(7)$ & $2(7)$ & $5(7)$ & 0.900 \\
\hline Intraoperative blood transfusion & $0(0-2)$ & $0(0-2)$ & $1(0-2)$ & 0.368 \\
\hline Intraoperative FFP & $3(0-5)$ & $3(0-4)$ & $3(0-6)$ & 0.463 \\
\hline \multicolumn{5}{|l|}{ Pathological examination } \\
\hline R1 resection, $n(\%)$ & $12(12)$ & $3(10)$ & $9(13)$ & 0.606 \\
\hline \multicolumn{5}{|l|}{ pT category, $n(\%)$} \\
\hline 1 & $7(7)$ & $2(7)$ & $5(7)$ & \multirow{4}{*}{0.678} \\
\hline 2 & $62(61)$ & $18(58)$ & $44(63)$ & \\
\hline 3 & $26(26)$ & $10(32)$ & $16(23)$ & \\
\hline 4 & $6(6)$ & $1(3)$ & $5(7)$ & \\
\hline
\end{tabular}


Table 1 (continued)

\begin{tabular}{|c|c|c|c|c|}
\hline Demographics & $\begin{array}{l}\text { Overall cohort } \\
(n=101)\end{array}$ & $\begin{array}{l}\text { High NFD } \\
(n=31)\end{array}$ & $\begin{array}{l}\text { Low NFD } \\
(n=70)\end{array}$ & $p$ value \\
\hline \multicolumn{5}{|l|}{ pN category } \\
\hline N1 & $39(39)$ & $10(32)$ & $29(41)$ & 0.383 \\
\hline \multicolumn{5}{|l|}{ Tumor grading, $n(\%)$} \\
\hline G1 & $6(6)$ & $2(7)$ & $4(6)$ & \multirow{2}{*}{0.099} \\
\hline $\mathrm{G} 4$ & $1(1)$ & 0 & $1(2)$ & \\
\hline MVI, $n(\%)$ & $71(73)$ & $4(13)$ & $21(32)$ & 0.100 \\
\hline LVI, $n(\%)$ & $21(22)$ & $7(23)$ & $14(22)$ & 0.938 \\
\hline PNI, $n(\%)$ & $66(81)$ & $18(82)$ & $48(80)$ & 0.854 \\
\hline \multicolumn{5}{|l|}{ Postoperative data } \\
\hline Clavien-Dindo I & $8(8)$ & $3(10)$ & $5(7)$ & \multirow{7}{*}{0.272} \\
\hline Clavien-Dindo II & $27(27)$ & $10(32)$ & $17(24)$ & \\
\hline Clavien-Dindo IIIa & $19(19)$ & $2(7)$ & $17(24)$ & \\
\hline Clavien-Dindo IIIb & $22(22)$ & $7(23)$ & $15(21)$ & \\
\hline Clavien-Dindo IVa & $7(7)$ & $3(10)$ & $4(6)$ & \\
\hline Clavien-Dindo IVb & $4(4)$ & 0 & $4(6)$ & \\
\hline Clavien-Dindo V & 0 & 0 & 0 & \\
\hline CCI & $35(21-50)$ & $23(8-54)$ & $39(20-49)$ & 0.124 \\
\hline \multicolumn{5}{|l|}{ Oncologic data } \\
\hline Adjuvant therapy & $23(23)$ & $6(19)$ & $17(25)$ & 0.562 \\
\hline Median RFS, months (95\% CI) & $37(18-56)$ & $83(34-132)$ & $24(13-35)$ & 0.004 \\
\hline Median CSS, months (95\% CI) & $49(29-69)$ & $90(48-132)$ & $33(19-47)$ & 0.006 \\
\hline
\end{tabular}

Data are presented as median and interquartile range if not noted otherwise. Bold numbers indicate statistical significance $(p<0.05)$. NFD, nerve fiber density; ALT, alanine aminotransferase; ASA, American Society of Anesthesiologists classification; AST, aspartate aminotransferase; CCI, comprehensive complication index; CSS, cancer-specific survival; EBD, endoscopic biliary drainage; FFP, fresh frozen plasma; GGT, gamma glutamyltransferase; INR, international normalized ratio; LVI, lymphovascular invasion; MVI, microvascular invasion; PBD, percutaneous biliary drainage; PNI, perineural invasion, RFS, recurrence-free survival; PVE, portal vein embolization.

Fig. 2. Oncological survival in pCCA. a CSS and OS in pCCA. The median CSS was 49 months (95\% CI: 29-69) and the median OS 33 months (95\% CI: 19-47), respectively. b RFS in pCCA. The median RFS was 37 months (95\% CI: 18-56). c PGP staining of pCCA with high NFD. Zoomed in image of the tumor with a lot of small nerves in the stroma between the tumor glands (blue arrows). On the zoomed in image of the routine HE staining, these small nerve fibers are not visible. These results occur in patients corresponding to the blue line in the Kaplan-Meier curve in $\mathbf{d}$ and $\mathbf{f}$ (high NFD). d CSS in pCCA stratified by NFD. The median CSS was 90 months (95\% CI: 48-132) in patients with high NFD compared to 33 months (95\% CI: 19-47) in patients with low NFD ( $p=0.006 \log$ rank). e PGP staining of pCCA with low NFD. Zoomed in image of the tumor without any small nerves in the stroma between the tumor glands. The zoomed in image of PGP shows a positive bigger nerve trunk with perineural invasion. These big nerve fibers are also easily recognized on the zoomed in routine HE staining. $f$ RFS in pCCA stratified by NFD. The median RFS was 83 months (95\%
CI: 34-132) in patients with high NFD compared to 24 months (95\% CI: 13-35) in patients with low NFD ( $p=0.004$ log rank). $\mathbf{g}$ CSS in pCCA stratified by NFD and pN category. The median CSS was 90 months (95\% CI: 57-123) in patients with high NFD and negative lymph nodes, 51 months (95\% CI: 38-64) in patients with either positive lymph nodes or low NFD but not both, and 24 months (95\% CI: 14-32) in patients with both positive lymph nodes and low NFD ( $p=0.001$ log rank). $\mathbf{h}$ RFS in pCCA stratified by NFD and pN category. The median RFS was 83 months $(95 \%$ CI: 42-124) in patients with high NFD and negative lymph nodes, 45 months (95\% CI: 8-82) in patients with either positive lymph nodes or low NFD but not both, and 10 months (95\% CI: 0-21) in patients with both positive lymph nodes and low NFD $(p=0.001$ log rank). CI, confidence interval; CSS, cancer-specific survival; RFS, recurrence-free survival; OS, overall survival; pCCA, perihilar cholangiocarcinoma; PGP, protein gene product 9.5; NFD, nerve fiber density.

(For figure see next page.) 


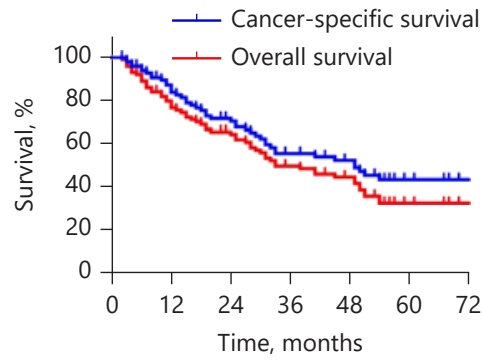

Numberst at risk $\begin{array}{lllllll}\text { CCS } 101 & 76 & 56 & 41 & 32 & 16 & 11\end{array}$

a $\begin{array}{lllllll}\text { OS } 101 & 76 & 56 & 41 & 32 & 16 & 11\end{array}$
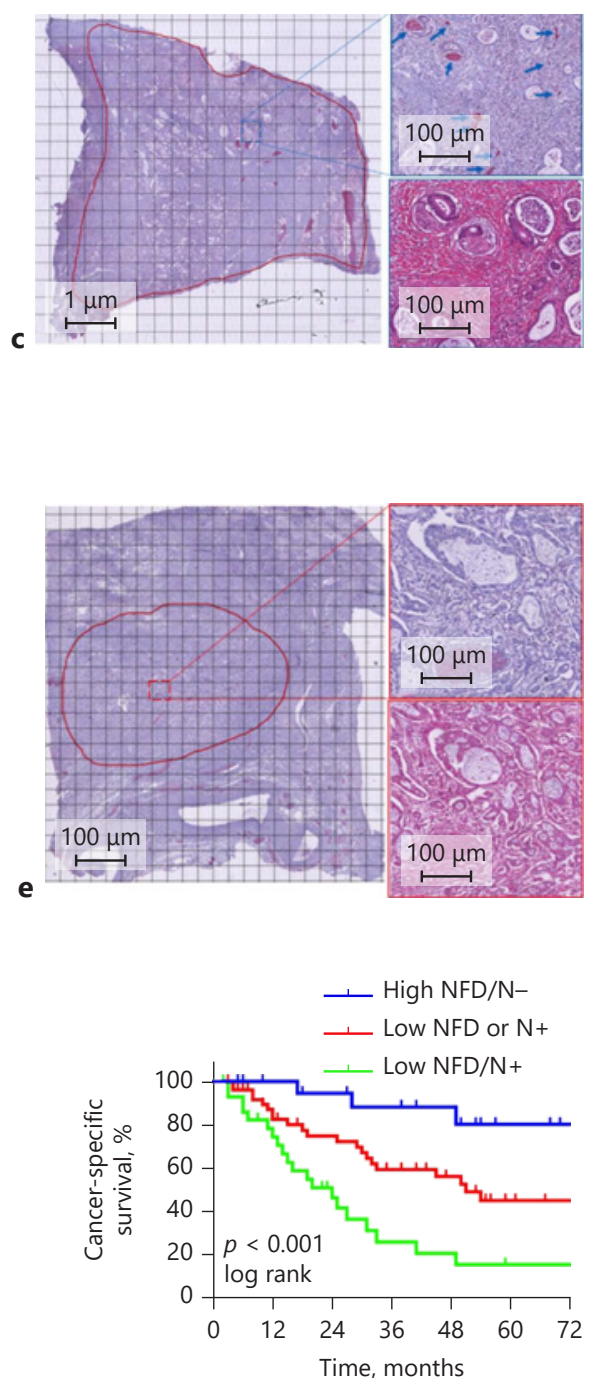

Numbers at risk

$\begin{array}{llllllll}\text { High NFD/N }- & 21 & 19 & 17 & 15 & 12 & 6 & 4\end{array}$ $\begin{array}{llllllll}\text { Low NFD or N+ } & 51 & 38 & 31 & 23 & 17 & 8 & 6\end{array}$

g Low NFD/N+ $\begin{array}{lllllll}29 & 20 & 11 & 6 & 5 & 3 & 2\end{array}$

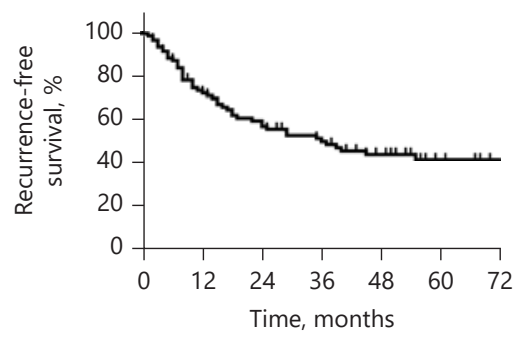

Number at risk

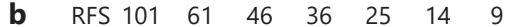

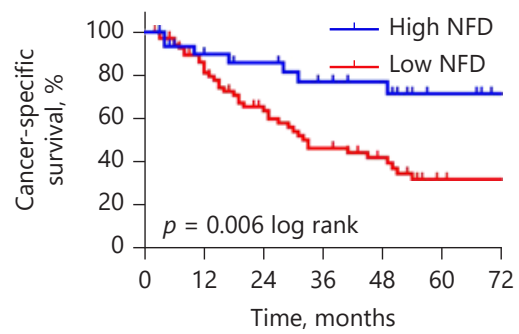

Number at risk

$\begin{array}{llllllll}\text { High NFD } & 31 & 24 & 22 & 17 & 15 & 8 & 5\end{array}$

$\begin{array}{llllllll}\text { d Low NFD } & 70 & 52 & 35 & 26 & 18 & 9 & 7\end{array}$

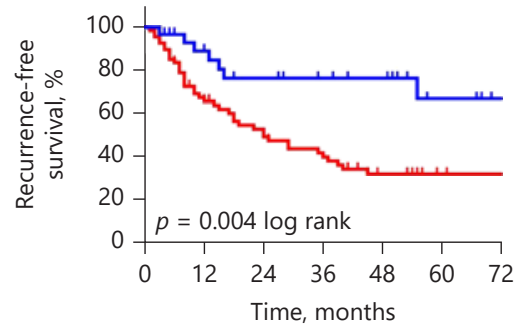

Number at risk

$\begin{array}{llllllll}\text { High NFD } & 31 & 22 & 18 & 15 & 13 & 7 & 4\end{array}$

$\begin{array}{llllllll}\text { f Low NFD } & 70 & 39 & 29 & 22 & 13 & 8 & 6\end{array}$

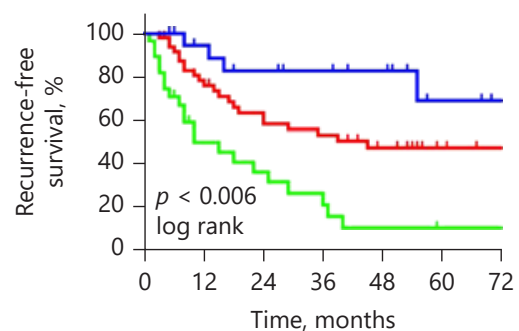

Numbers at risk

$\begin{array}{llllllll}\text { High NFD/N }- & 21 & 17 & 14 & 12 & 10 & 5 & 3\end{array}$

Low NFD or N+ $\begin{array}{lllllll}51 & 34 & 25 & 22 & 14 & 8 & 6\end{array}$

h Low NFD/N+ $\begin{array}{lllllll}29 & 13 & 9 & 5 & 3 & 2 & 2\end{array}$

2

Nerve Fiber Density in Perihilar Cholangiocarcinoma 


\section{Results}

\section{Patient Cohort}

The study cohort consisted of 68 men $(67 \%)$ and 33 women $(33 \%)$ with a median age of 68 years. The majority of patients presented with Bismuth type III (55\%, $55 / 101)$ or IV $(32 \%, 32 / 101)$ tumors and were assessed as ASA (American Society of Anesthesiologists classification) III or higher $(53 \%, 53 / 101)$. Neoadjuvant therapy was applied in a small number of patients $(4 \%$, $4 / 101$ ), while preoperative portal vein embolization was carried out in a significant proportion of patients $(43 \%$, 43/101). Mandatory portal vein resection and reconstruction was carried out in every patient (101/101), while additional arterial reconstruction was necessary in $7 \%(7 / 101)$. Also, the concomitant resection of the pancreatic head was needed in $6 \%(6 / 101)$ of the patients to achieve clear tumor margins. Accordingly, R1 resection was confirmed in $12 \%(12 / 101)$ of the overall cohort. Major complications after surgery were frequently observed with $33 \%$ (33/101) of the patients presenting with complications $\geq$ Clavien-Dindo IIIb. Cases with perioperative mortality were excluded from the analysis as stated above. Further demographic and clinicopathological details of the cohort are outlined in Table 1 .

\section{Group Categorization and Comparative Analysis with} Respect to NFD

A receiver operating characteristic analysis evaluating the total number of nerve fibers for patients who survived at least 4 years versus patients who died during follow-up was conducted. The corresponding area under the curve was 0.618 (95\% confidence interval [CI]: 0.480-0.756). A cutoff value for NFD was determined with respect to optimized accuracy and equal weight for sensitivity and specificity errors ( $<10$ nerve fibers and $\geq 10$ nerve fibers). Using the established cutoff value, the median CSS was 90 months in patients with high NFD ( $\geq 10$ nerve fibers) and 33 months in patients with low NFD $(<10$ nerve fibers, $p=0.006$ log rank).

A comparative group analysis regarding NFD was further carried out between patients with high NFD $(n=31)$ and low NFD $(n=70)$. Extensive group comparisons revealed no significant differences in clinical characteristics expect a longer median hospitalization time in the low NFD group (14 vs. 21 days, $p=0.017$ ). Of note, no statistical differences in $\mathrm{pT}$ category $(p=0.678), \mathrm{pN}$ category $(p=0.383)$, tumor grading $(p=0.099), \mathrm{LVI}(p=0.938)$, microvascular invasion (MVI, $p=0.100)$, and PNI ( $p=$
$0.854)$ were observed between the groups. However, the median CSS (90 months [95\% CI: 48-132] vs. 33 months [95\% CI: 19-47], $p=0.006$ log rank) and the median RFS (83 months [95\% CI: $34-132$ ] vs. 24 months [95\% CI: 1335], $p=0.004 \log$ rank) were significantly longer in patients with high NFD compared to patients with low NFD. More details regarding the group comparisons are presented in Table 1.

\section{Survival Analysis}

After a median follow-up of 53 months, the median CSS of the whole cohort was 49 months (95\% CI: 2969), the median OS 33 months (95\% CI: 19-47), and the median RFS 37 months (95\% CI: 18-56, Fig. 2a, b). A Kaplan-Meier analysis with respect to NFD showed a median CSS of 90 months (95\% CI: 48-132, 3-year CSS $=77 \%$, 5-year CSS $=72 \%)$ in patients with high NFD compared to 33 months (95\% CI: 19-47, 3-year CSS = $46 \%, 5$-year CSS $=32 \%)$ in patients with low NFD ( $p=$ 0.006 log rank, Fig. 2c). Further, RFS was significantly lower in patients with low NFD (24 months [95\% CI: 13-35]) compared to patients with high NFD (83 months [95\% CI: 34-132], $p=0.004$ log rank, Fig. 2d). Interestingly, in a subsequent Kaplan-Meier analysis, the combination of NFD with nodal status resulted in a median CSS of 90 months (95\% CI: 57-123, 3-year CSS $=88 \%, 5$-year CSS $=80 \%)$ in patients with high NFD and negative lymph nodes, 51 months (95\% CI: 38-64, 3 -year CSS $=59 \%$, 5-year CSS $=45 \%)$ in patients with either positive lymph nodes or low NFD but not both, and 24 months (95\% CI: $14-32,3$-year CSS $=26 \%$, 5 -year CSS $=16 \%$ ) in patients with both positive lymph nodes and low NFD ( $p=0.001$ log rank, Fig. 2e). Accordingly, the median RFS was 83 months (95\% CI: $42-$ 124) in patients with high NFD and negative lymph nodes, 45 months (95\% CI: 8-82) in patients with either positive lymph nodes or low NFD, and 10 months (95\% CI: 0-21) in patients with both positive lymph nodes and low NFD ( $p=0.001$ log rank, Fig. 2f).

\section{Cox Regression Analysis}

In univariate analysis, intraoperative blood $(p=$ $0.011)$ and FFP transfusion $(p=0.010)$, R1 resection $(p=0.019)$, nodal status $(p=0.002)$, tumor grading $(p=0.004)$, MVI $(p=0.022)$, LVI $(p=0.026)$, and NFD $(p=0.009)$ were significantly associated with CSS. All variables showing $p$ value $<0.05$ were included in a multivariable Cox regression model. Here, intraoperative FFP transfusion $(\mathrm{HR}=2.90, p=0.004)$, nodal status $(\mathrm{HR}=2.84, p=0.001)$, and $\mathrm{NFD}(\mathrm{HR}=0.41, p=0.024)$ 
Table 2. Univariate and multivariable analysis of CSS in pCCA

\begin{tabular}{|c|c|c|c|c|}
\hline & \multicolumn{2}{|l|}{ Univariate analysis } & \multicolumn{2}{|c|}{ Multivariable analysis } \\
\hline & HR (95\% CI) & $p$ value & HR (95\% CI) & $p$ value \\
\hline \multicolumn{5}{|l|}{ Demographics } \\
\hline Sex $($ male $=1)$ & $1.28(0.71-2.29)$ & 0.411 & & \\
\hline Age $(\leq 65$ years $=1)$ & $1.13(0.64-2.00)$ & 0.683 & & \\
\hline $\mathrm{BMI}\left(\leq 25 \mathrm{~kg} / \mathrm{m}^{2}=1\right)$ & $1.17(0.66-2.06)$ & 0.591 & & \\
\hline $\operatorname{PVE}($ no $=1)$ & $1.01(0.57-1.80)$ & 0.972 & & \\
\hline ASA $(I / I I=1)$ & $1.27(0.72-2.27)$ & 0.411 & & \\
\hline Preoperative cholangitis $($ no $=1$ ) & $0.82(0.44-1.53)$ & 0.526 & & \\
\hline $\operatorname{EBD}($ no $=1)$ & $1.29(0.60-2.76)$ & 0.515 & & \\
\hline $\operatorname{PBD}($ no $=1)$ & $0.95(0.50-1.84)$ & 0.889 & & \\
\hline \multicolumn{5}{|l|}{ Clinical chemistry } \\
\hline $\operatorname{Albumin}(\leq 40 \mathrm{~g} / \mathrm{L}=1)$ & $0.68(0.36-1.28)$ & 0.238 & & \\
\hline $\operatorname{AST}(\leq 40 \mathrm{U} / \mathrm{L}=1)$ & $1.34(0.72-2.48)$ & 0.739 & & \\
\hline $\operatorname{ALT}(\leq 40 \mathrm{U} / \mathrm{L}=1)$ & $0.87(0.40-1.93)$ & 0.354 & & \\
\hline $\mathrm{GGT}(\leq 400 \mathrm{U} / \mathrm{L}=1)$ & $1.20(0.66-2.20)$ & 0.555 & & \\
\hline Bilirubin $(\leq 1 \mathrm{mg} / \mathrm{dL}=1)$ & $1.26(0.72-2.23)$ & 0.423 & & \\
\hline Alkaline phosphatase $(\leq 250 \mathrm{U} / \mathrm{L}=1)$ & $1.01(0.56-1.86)$ & 0.964 & & \\
\hline Platelet count $(\leq 300 / \mathrm{nL}=1)$ & $1.08(0.59-1.96)$ & 0.813 & & \\
\hline $\operatorname{INR}(\leq 1=1)$ & $1.57(0.84-2.95)$ & 0.162 & & \\
\hline $\operatorname{Hemoglobin}(\leq 13 \mathrm{~g} / \mathrm{dL}=1)$ & $0.63(0.34-0.1 .17)$ & 0.144 & & \\
\hline $\mathrm{CRP}, \mathrm{mg} / \mathrm{L}(\leq 10 \mathrm{mg} / \mathrm{L}=1)$ & $1.08(0.59-1.95)$ & 0.813 & & \\
\hline \multicolumn{5}{|l|}{ Operative data } \\
\hline Operative time $(\leq 360 \mathrm{~min}=1)$ & $1.68(0.87-3.27)$ & 0.125 & & \\
\hline Type of hepatectomy & & & & \\
\hline Right-sided hepatectomy & 1 & 0.965 & & \\
\hline Left-sided hepatectomy & $0.97(0.53-1.74)$ & 0.907 & & \\
\hline Blood transfusion $($ no $=1)$ & $2.11(1.19-3.75)$ & 0.011 & Excluded & \\
\hline FFP transfusion $($ no $=1)$ & $2.38(1.23-4.61)$ & 0.010 & $2.90(1.39-6.02)$ & 0.004 \\
\hline Arterial resection $($ no $=1)$ & $1.85(0.66-5.19)$ & 0.242 & & \\
\hline \multicolumn{5}{|l|}{ Pathological data } \\
\hline $\mathrm{R} 1$ resection $($ no $=1)$ & $2.42(1.16-5.06)$ & 0.019 & Excluded & \\
\hline pT category $(\mathrm{T} 1 / \mathrm{T} 2=1)$ & $1.53(0.85-2.78)$ & 0.159 & & \\
\hline $\mathrm{pN}$ category $(\mathrm{N} 0=1)$ & $2.52(1.32-4.47)$ & 0.002 & $2.84(1.52-5.31)$ & 0.001 \\
\hline Tumor grading $(\mathrm{G} 1 / \mathrm{G} 2=1)$ & $2.86(1.39-5.88)$ & 0.004 & Excluded & \\
\hline $\operatorname{MVI}(\mathrm{no}=1)$ & $2.10(1.16-3.96)$ & 0.022 & Excluded & \\
\hline LVI $($ no $=1)$ & $2.14(1.09-4.18)$ & 0.026 & Excluded & \\
\hline PNI $($ no $=1)$ & $1.72(0.67-4.42)$ & 0.260 & & \\
\hline NFD $($ low = 1) & $0.37(0.18-0.78)$ & 0.009 & $0.41(0.19-0.89)$ & 0.024 \\
\hline \multicolumn{5}{|l|}{ Oncological data } \\
\hline Neoadjuvant therapy $($ no $=1)$ & $0.58(0.08-4.21)$ & 0.589 & & \\
\hline Adjuvant therapy $($ yes $=1)$ & $1.52(0.78-2.97)$ & 0.219 & & \\
\hline
\end{tabular}

Various parameters are associated with overall survival. Bold numbers indicate statistical significance $(p<$ 0.05). CSS, cancer-specific survival; pCCA, perihilar cholangiocarcinoma; ALT, alanine aminotransferase; ASA, American Society of Anesthesiologists classification; AST, aspartate aminotransferase; CCI, comprehensive complication index; CRP, C-reactive protein; EBD, endoscopic biliary drainage; FFP, fresh frozen plasma; GGT, gamma glutamyltransferase; INR, international normalized ratio; LVI, lymphovascular invasion; MVI, microvascular invasion; NFD, nerve fiber density; PBD, percutaneous biliary drainage; PNI, perineural invasion; PVE, portal vein embolization.

were identified as independent predictors of CSS (Table 2).

In univariate analysis, intraoperative blood $(p=0.034)$ and FFP transfusion $(p=0.009)$, R1 resection $(p=0.006)$, nodal status ( $p=0.003)$, tumor grading $(p=0.001)$, MVI $(p=0.008)$, LVI $(p=0.022)$, NFD $(p=0.007)$, and adjuvant therapy $(p=0.028)$ showed significant associations with RFS. In the corresponding multivariable Cox re- 
Table 3. Univariate and multivariable analysis of RFS in pCCA

\begin{tabular}{|c|c|c|c|c|}
\hline & \multicolumn{2}{|c|}{ Univariate analysis } & \multicolumn{2}{|c|}{ Multivariable analysis } \\
\hline & HR (95\% CI) & $p$ value & HR (95\% CI) & $p$ value \\
\hline \multicolumn{5}{|l|}{ Demographics } \\
\hline Sex $($ male $=1)$ & $1.12(0.62-2.02)$ & 0.704 & & \\
\hline Age $(\leq 65$ years $=1)$ & $0.89(0.51-1.56)$ & 0.691 & & \\
\hline $\mathrm{BMI}\left(\leq 25 \mathrm{~kg} / \mathrm{m}^{2}=1\right)$ & $1.46(0.83-2.58)$ & 0.189 & & \\
\hline $\operatorname{PVE}($ no $=1)$ & $1.23(0.70-2.16)$ & 0.478 & & \\
\hline $\operatorname{ASA}(I / I I=1)$ & $1.00(0.57-1.75)$ & 0.990 & & \\
\hline Preoperative cholangitis $($ no $=1$ ) & $1.71(0.94-3.09)$ & 0.077 & & \\
\hline $\operatorname{EBD}($ no $=1)$ & $1.23(0.60-2.54)$ & 0.576 & & \\
\hline $\operatorname{PBD}($ no $=1)$ & $1.12(0.58-2.14)$ & 0.741 & & \\
\hline \multicolumn{5}{|l|}{ Clinical chemistry } \\
\hline Albumin $(\leq 40 \mathrm{~g} / \mathrm{L}=1)$ & $0.61(0.33-1.13)$ & 0.114 & & \\
\hline $\operatorname{AST}(\leq 40 \mathrm{U} / \mathrm{L}=1)$ & $1.49(0.81-2.74)$ & 0.200 & & \\
\hline $\operatorname{ALT}(\leq 40 \mathrm{U} / \mathrm{L}=1)$ & $1.00(0.46-2.17)$ & 0.997 & & \\
\hline $\operatorname{GGT}(\leq 400 \mathrm{U} / \mathrm{L}=1)$ & $1.25(0.69-2.28)$ & 0.463 & & \\
\hline Bilirubin $(\leq 1 \mathrm{mg} / \mathrm{dL}=1)$ & $1.26(0.72-2.23)$ & 0.423 & & \\
\hline Alkaline phosphatase $(\leq 250 \mathrm{U} / \mathrm{L}=1)$ & $0.96(0.53-1.75)$ & 0.901 & & \\
\hline Platelet count $(\leq 300 / \mathrm{nL}=1)$ & $1.26(0.68-2.31)$ & 0.464 & & \\
\hline $\operatorname{INR}(\leq 1=1)$ & $1.75(0.94-3.27)$ & 0.077 & & \\
\hline Hemoglobin $(\leq 13 \mathrm{~g} / \mathrm{dL}=1)$ & $0.56(0.30-1.05)$ & 0.069 & & \\
\hline $\mathrm{CRP}, \mathrm{mg} / \mathrm{L}(\leq 10 \mathrm{mg} / \mathrm{L}=1)$ & $1.34(0.74-2.43)$ & 0.343 & & \\
\hline \multicolumn{5}{|l|}{ Operative data } \\
\hline Operative time $(\leq 360 \mathrm{~min}=1)$ & $1.67(0.85-3.27)$ & 0.136 & & \\
\hline Type of hepatectomy & & & & \\
\hline Right-sided hepatectomy & 1 & 0.466 & & \\
\hline Left-sided hepatectomy & $0.81(0.45-1.44)$ & & & \\
\hline Blood transfusion $($ no $=1)$ & $1.84(1.05-3.24)$ & 0.034 & Excluded & \\
\hline FFP transfusion $($ no $=1)$ & $2.33(1.23-4.40)$ & 0.009 & $3.10(1.42-6.30)$ & 0.002 \\
\hline Arterial resection $($ no $=1)$ & $0.88(0.21-3.67)$ & 0.862 & & \\
\hline \multicolumn{5}{|l|}{ Pathological data } \\
\hline $\mathrm{R} 1$ resection $($ no $=1)$ & $2.69(1.33-5.44)$ & 0.006 & Excluded & \\
\hline pT category $(\mathrm{T} 1 / \mathrm{T} 2=1)$ & $1.64(0.92-2.94)$ & 0.095 & & \\
\hline pN category $(\mathrm{N} 0=1)$ & $2.33(1.32-4.10)$ & 0.003 & $2.92(1.55-5.48)$ & 0.001 \\
\hline Tumor grading (G1/G2 = 1) & $3.51(1.82-6.76)$ & 0.001 & Excluded & \\
\hline $\operatorname{MVI}($ no $=1)$ & $2.27(1.23-4.19)$ & 0.008 & $1.98(1.01-3.89)$ & 0.048 \\
\hline $\operatorname{LVI}($ no $=1)$ & $2.13(1.11-4.08)$ & 0.022 & Excluded & \\
\hline PNI $($ no $=1)$ & $2.41(0.86-6.80)$ & 0.096 & & \\
\hline NFD $($ low = 1) & $0.35(0.16-0.75)$ & 0.007 & $0.42(0.19-0.93)$ & 0.031 \\
\hline \multicolumn{5}{|l|}{ Oncological data } \\
\hline Neoadjuvant therapy $($ no $=1)$ & $0.49(0.07-3.55)$ & 0.479 & & \\
\hline Adjuvant therapy $($ yes $=1)$ & $0.50(0.27-0.93)$ & 0.028 & Excluded & \\
\hline
\end{tabular}

Various parameters are associated with disease-free survival. Bold numbers indicate statistical significance $(p<0.05)$. RFS, recurrence-free survival; pCCA, perihilar cholangiocarcinoma; ALT, alanine aminotransferase; ASA, American Society of Anesthesiologists classification; AST, aspartate aminotransferase; CCI, comprehensive complication index; CRP, C-reactive protein; EBD, endoscopic biliary drainage; FFP, fresh frozen plasma; GGT, gamma glutamyltransferase; INR, international normalized ratio; LVI, lymphovascular invasion; MVI, microvascular invasion; NFD, nerve fiber density; PBD, percutaneous biliary drainage; PNI, perineural invasion; PVE, portal vein embolization.

gression model, intraoperative FFP transfusion ( $\mathrm{HR}=$ $3.10, p=0.002)$, nodal status ( $\mathrm{HR}=2.92, p=0.001)$, MVI $(\mathrm{HR}=1.98, p=0.048)$ and NFD $(\mathrm{HR}=0.42, p=0.031)$ were identified as independent predictors of RFS (Table 3).

\section{Analysis of Nerve Fiber Origin}

To further investigate the origin of the counted NFD, immunohistochemistry was carried out in a representative subset of patients $(n=20)$. Here, small nerve fibers counted to assess NFD were stained positive for vasoac- 
Fig. 3. Origin of the nerve fibers. Schematic overview of tissue with cancers cells invading the nerve. a Routine $\mathrm{HE}$ staining showing perineural invasion of cancer cells invading a large nerve trunk (red arrow). b PGP immunohistochemistry staining being expressed in this cancer-invaded nerve (yellow arrow). NPY immunohistochemistry (sympathetic origin) is expressed (c), and VIP immunohistochemistry (parasympathetic) is not expressed in this large nerve trunk (d). Schematic overview of tissue with cancer cells not invading the nerve. e Routine HE staining showing the yellow arrow to the localization of the small nerve fibers that are not visible on the HE staining. $\mathbf{f}$ Those small nerve fibers stain positive in the PGP immunohistochemistry. $g$ Negative staining of these small nerve fibers in the VPN immunohistochemistry. h Yellow arrow points to the positive staining in the VIP immunohistochemistry of these small nerve fibers. NPY, neuropeptide Y; VIP, vasoactive intestinal peptide.

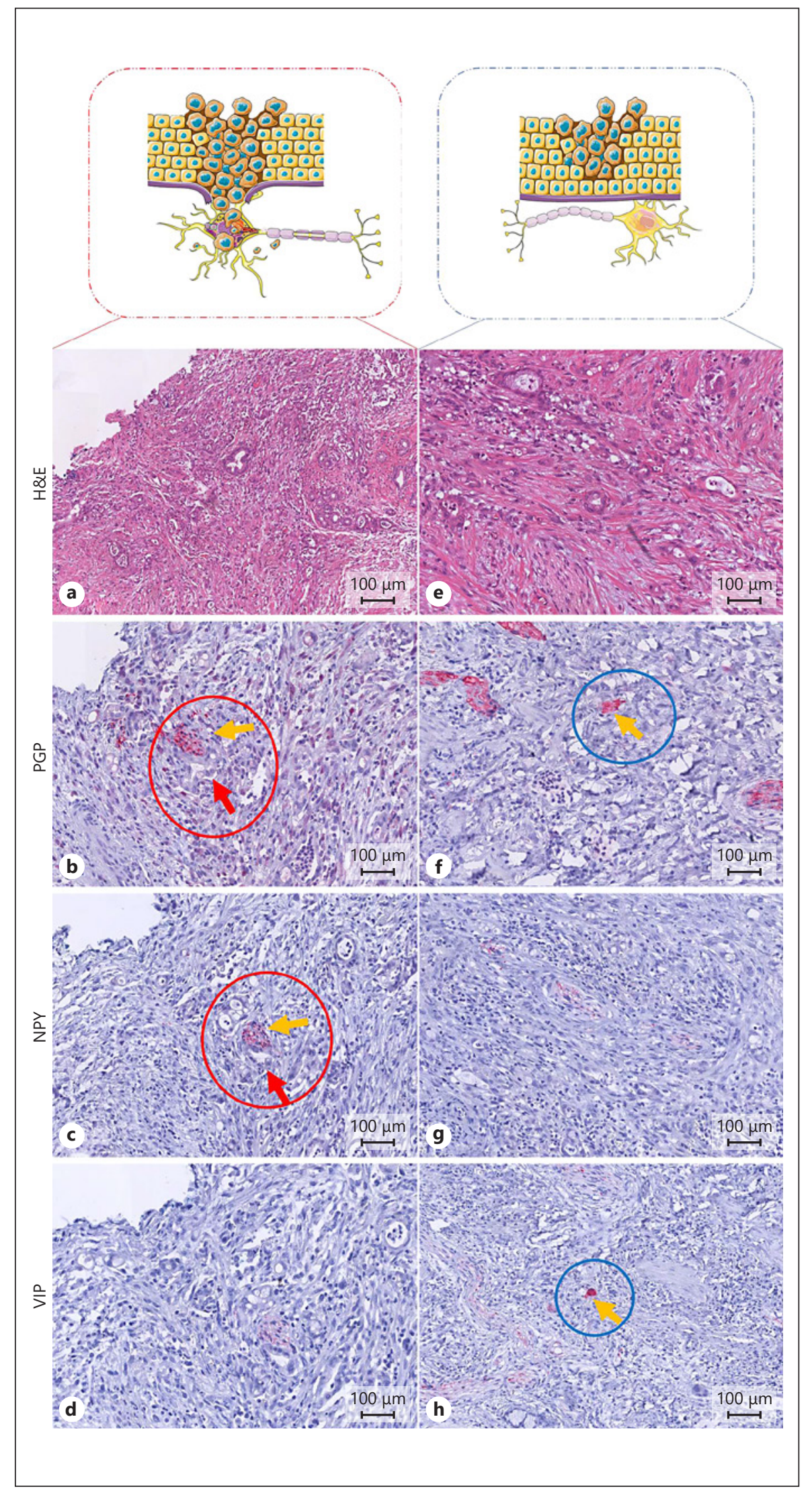

Nerve Fiber Density in Perihilar Cholangiocarcinoma
Liver Cancer 2021;10:260-274

DOI: $10.1159 / 000515303$ 
tive intestinal peptide (to indicate parasympathetic origin) but negative for NPY (to indicate sympathetic origin). The larger pre-existing nerve fibers which also stained positive in the PGP marker showed expression of the NPY marker, and those nerve fibers were observed to be invaded by tumor cells (Fig. 3).

\section{Discussion and Conclusion}

Radical surgery with lymphadenectomy represents the current gold standard therapy of resectable pCCA [15]. However, oncological outcome remains heterogeneous after curative-intent surgery with some patients displaying long-term survival while other individuals suffer from early tumor recurrence [3]. Therefore, the identification of prognostic factors is of clinical and academic significance as it provides implications for clinical management and may give insights in the underlying tumor biology of the disease [24]. In this large European cohort of pCCA patients, we identified NFD as a novel and important prognostic biomarker for oncological outcome in these patients. The combination of NFD and nodal status demonstrated an excellent ability to stratify pCCA regarding their oncological prognosis after curative-intent surgery for pCCA.

CCA is considered to be a "neurotopic" cancer with a high frequency of PNI which is associated with impaired outcomes $[17,25]$. The biliary tree is surrounded by a complex nerve fiber network being the basis for an intense crosstalk between nerve fibers and tumor cells as well as other parts of the TME, for example, immune cells or cancer-associated fibroblasts [26-28]. Neurotransmitters are known to directly interact with the cancer cells, and conversely cancer cells release neurotransmitters activating receptors on the nerve fibers [29-32]. Intratumor cancer-associated fibroblasts are remodeling the extracellular matrix and leading to a production of hyaluronic acid, fibronectin, and collagen. Those extracellular matrix components are known to influence neuron growth $[26,27]$. Also, the colocalization and interaction of immune cells and nerve fibers drive tissue protection and play a critical immune-regulatory role [28]. In this study, we have further identified the origin of these nerve fibers to be parasympathetic and specifically those nerve fibers predict a better outcome. Of note, from a clinical point of view, NFD has already been investigated in various other malignancies, for example, gastric and colorectal carcinomas $[33,34]$. Here, high NFD has been associated with a higher pathological staging and subsequently decreased survival. Interestingly, Iwasaki et al. [19] have recently reported on the role of NFD in PDAC and found an inverse relationship with low NFD being independently associated with reduced survival after surgical resection. This observation is in line with our present findings, identifying low NFD as an important predictor of inferior CSS in pCCA. In our study, we were able to demonstrate a 5 -year CSS of $72 \%$ in patients with high NFD compared to a 5 -year CSS of $32 \%$ in patients with low NFD (Fig. 2c).

The underlying mechanisms of this clinical observation are yet to be explored. Iwasaki et al. [19] demonstrated a correlation of lower NFD to a higher tumor grade in PDAC patients suggesting a biologically more aggressive disease. In our group comparison, we observed the tendency for a higher tumor grading in the low NFD group which did not reach statistical significance (Table 1). Nonetheless, whether low NFD tumors represent a more aggressive tumor type per se or are just more prone to be resistant to standard treatment cannot be explored in our setting. However, these data suggest a major oncological role of NFD in pCCA which warrants further basic research and potentially novel clinical approaches to patients with low NFD tumors.

The prognostic role of lymph node metastases in pCCA is abundantly discussed elsewhere [3, 35-38]. In line with these previous observations, nodal status was also of major predictive value in our multivariable analysis of oncological outcome. As nodal status was not associated with NFD in our cohort and the combination of both major oncological predictors might therefore be of particular value, we created 3 distinct subgroups by combining NFD and $\mathrm{pN}$ category as a next step in our analysis. Here, patients with high NFD and no nodal metastases showed a median CSS of 90 months compared to 51 months in patients with either positive lymph nodes or low NFD and 24 months in patients displaying both high risk features (Fig. 2e). This statistically significant observation translates into an 80\% 5-year CSS in the low-risk, $45 \%$ 5-year CSS in the median-risk, and 16\% 5-year CSS in the high-risk subcohorts of our analysis. An interesting observation was the particularly compelling outcome of the abovementioned low-risk cohort (high NFD and N-), while our median-risk group (low NFD or N+) is in line with previous reports and the high-risk group (low NFD and $\mathrm{N}+$ ) below the commonly reported outcome figures. This underlines the oncological role of NFD in pCCA [1$9,15]$. As such, the combination of NFD (which is assessable by inexpensive measures) and nodal status (which is routinely provided by the pathology report) does provide an easily applicable risk stratification for pCCA patients undergoing curative-intent surgery. 
To further investigate the counted NFD, we conducted immunohistochemistry and identified the small nerve fibers to be of parasympathetic origin. This finding is novel and interesting as the role of the parasympathetic nervous system in cancer initiation and progression is not fully understood. A potential inhibitory effect of the parasympathetic nervous system on cancer initiation was historically observed in patients who underwent vagotomy as a treatment for gastric ulcers and subsequently displayed a higher incidence for gastric cancer [39]. A recent study investigated the impact of subdiaphragmatic vagotomy in a murine PDAC model. Here, vagotomy increased tumor growth and impaired survival in this mouse model which was partly explained by increased expression of tumor necrosis factor alpha in tumor tissue [40]. Recently, Kamiya et al. [41] introduced an adeno-associated virus vector enabling the stimulation or inhibition of parasympathetic and sympathetic nerve fibers localized in the tumor tissue. In a xenograft model, they observed a decreased progression of the primary tumors and attenuation of the development of distant metastases after increasing parasympathetic neurotransmission. Additionally, the vector also showed antitumor activity in a chemically induced model of breast cancer $[41,42]$. While the investigation of the mechanism of our observation regarding the protective effect of parasympathetic nerve fibers is beyond the scope of our clinically oriented analysis, these observations provide further evidence for the significant interaction of the parasympathetic nervous system and cancer cells and warrants further research. In pancreatic cancer, our team and another group have reported that a low NFD indicates a poor survival $[19,43]$. Observational studies have shown differences in the role of an increased sympathetic innervation of tumors. For prostate cancer [44], breast cancer [41], and hepatocellular carcinoma [45], the presence of sympathetic nerve fibers was indicative for cancer progression. For gastric cancer [46] and colorectal cancer [47], the opposite has been described, and low sympathetic fiber density was associated with progression. This dual role of nerve fibers and their exact origin in pCCA are important research questions of ongoing studies of our group.

Tumor recurrence remains the major problem in pCCA patients who underwent curative-intent surgery $[48,49]$. Local recurrence is commonly diagnosed concomitantly with a stenosis at the surgically performed hepaticojejunostomy resulting in recurrent cholangitis and life-threating biliary sepsis [20]. In contrast, survival after metastatic recurrence is usually determined by the limited response and resistance to chemotherapy resulting in

Nerve Fiber Density in Perihilar

Cholangiocarcinoma early disease progression and associated fatal outcome [15]. These limitations in the treatment of tumor relapse were also observed in our cohort with most of the individuals experiencing tumor recurrence (median RFS 37 months) deceasing shortly after the diagnosis (median CSS 49 months). This close relationship between RFS and CSS also explains our finding that NFD is also associated with RFS in multivariable analysis.

Interestingly, adjuvant therapy was a risk factor for RFS in univariate analysis. This might be explained by our historic approach to apply adjuvant therapy in patients with a high risk for tumor recurrence (e.g., positive nodal status or R1 resection) in the early study period [50,51]. From 2018, patients with adequate performance status were subjected to adjuvant capecitabine-based therapy or referred for inclusion to the currently recruiting ACCTICA trial (Adjuvant Chemotherapy With Gemcitabine and Cisplatin Compared to Standard of Care After Curative Intent Resection of Biliary Tract Cancer, NCT02170090) in all cases if the patient was willing to participate in a clinical trial $[23,52]$.

Unfortunately, NFD cannot be assessed prior to surgery and is therefore not available for preoperative patient selection. However, NFD displayed a good prognostic ability for recurrence and reduced survival after surgery and might therefore be used for postoperative risk stratification. Based on our data, one could speculate that low NFD is associated with a biologically more aggressive tumor which might benefit from adjuvant therapy after surgery. Adjuvant therapy in biliary tract cancer is considered the standard of care in Europe after the results of the BILCAP trial but is not taken by every patient as the beneficial effects regarding survival appear marginal [23]. In other countries, patients are selected for adjuvant therapies if they display high-risk features as positive lymph nodes or residual tumor [53]. In this context, NFD could be seen as a high-risk feature similar to nodal status. Further, patients with low NFD should be closely monitored for signs of disease relapse irrespective of adjuvant therapy in order to facilitate an early treatment in case of recurrence.

For our statistical approach, we decided to exclude patients with perioperative mortality from our analysis and reported oncological outcome in terms of CSS. Liver resection for pCCA is a high-risk procedure with a perioperative mortality up to $15 \%[54,55]$. Also, as previously reported by our group, our local policies comprise portal vein reconstruction in every patient and arterial reconstruction and/ or concomitant resection of the pancreatic head on demand within a cohort characterized by high ASA patients 
and complex tumors in terms of Bismuth staging [3, 9]. As the aim of this study was to unravel the prognostic role of the pathological parameter NFD, the exclusion of perioperative mortality appears reasonable. Also, our group comparison with respect to NFD showed no statistical difference between patients with low and high NFD regarding perioperative characteristics (Table 1). Further, CSS instead of OS was used to evaluate the oncological importance of NFD. Our monocentric cohort undergoes a detailed follow-up allowing to report CSS. Due to our aggressive surgical approach to the disease in general, our cohort comprises a median age of 68 years (range $49-80$ years) and significant comorbidities ( $>50 \%$ patients assessed ASA III or higher). Therefore, a significant proportion of patients deceased due to cancer-unassociated circumstances, for example, cardiovascular events, secondary malignancies, or late surgical complications such as obstructive ileus. As our sample size is limited due to the monocentric nature of our study and might therefore not compensate these effects from a statistical point of view, CSS appeared more appropriate to investigate the true oncological effect of NFD on long-term outcome.

As with all oncological outcome studies, our analysis has some potential limitations. All patients included in this study were treated at a single institution reflecting the authors' individual surgical approach, and all data were collected and analyzed retrospectively. This retrospective nature of our study resulted in some missing data which would have been interesting in the context of the oncological analysis, for example, CA19-9. The monocentric approach also results in a limited sample size which did not allow to split the cohort into a training and validation set. Therefore, our findings warrant further validation in an independent dataset.
Notwithstanding the abovementioned limitations, we have identified NFD as a novel and important prognostic biomarker in pCCA patients. NFD alone and in combination with nodal status in particular allows to stratify pCCA patients in terms of oncological outcome after curative-intent surgery. Larger, multicenter studies are needed to confirm and validate our findings.

\section{Statement of Ethics}

The study was conducted in accordance with the requirements of the Institutional Review Board of the RWTH-Aachen University (EK 106/18), the current version of the Declaration of Helsinki, and the good clinical practice guidelines (ICH-GCP). A written informed consent was obtained from all patients.

\section{Conflict of Interest Statement}

The authors have no conflicts of interest to declare.

\section{Funding Sources}

X.T. was funded by the China Scholarship Council (CSC Grant No. 201806210074).

\section{Author Contributions}

L.R.H. and J.B. designed the study. L.R.H. and J.B. were responsible for data analysis and interpretation. X.T. and L.R.H. annotated all tumor samples manually. J.B. and L.R.H. performed statistical analysis and interpretation. L.R.H., G.W., U.P.N., F.U., J.K., C.C., Z.C., and S.S. contributed to the first drafted initial manuscript. C.C. and L.R.H. provided tumor blocks and histology expertise. U.P.N. and F.U. provided infrastructure and supervised the study. All authors contributed to the data analysis and to writing the manuscript.

\section{References}

1 Neumann UP, Schmeding M. Role of surgery in cholangiocarcinoma: from resection to transplantation. Best Pract Res Clin Gastroenterol. 2015 Apr;29(2):295-308.

2 Torre LA, Bray F, Siegel RL, Ferlay J, LortetTieulent J, Jemal A. Global cancer statistics, 2012. CA Cancer J Clin. 2015 Mar;65(2):87108.

3 Lurje G, Bednarsch J, Czigany Z, Lurje I, Schlebusch IK, Boecker J, et al. The prognostic role of lymphovascular invasion and lymph node metastasis in perihilar and intrahepatic cholangiocarcinoma. Eur J Surg Oncol. 2019 Aug;45(8):1468-78.
4 Miyazaki M, Ito H, Nakagawa K, Ambiru S, Shimizu H, Shimizu Y, et al Aggressive surgical approaches to hilar cholangiocarcinoma: hepatic or local resection? Surgery. 1998 Feb; 123(2):131-6.

5 Neuhaus P, Jonas S, Bechstein WO, Lohmann R, Radke C, Kling N, et al. Extended resections for hilar cholangiocarcinoma. Ann Surg. 1999 Dec;230(6):808-19; discussion 19.

6 Becker T, Lehner F, Bektas H, Meyer A, Lück $\mathrm{R}$, Nashan B, et al. [Surgical treatment for hilar cholangiocarcinoma (Klatskin's tumor)]. Zentralbl Chir. 2003 Nov;128(11):928-35.
7 Petrowsky H, Wildbrett P, Husarik DB, Hany TF, Tam S, Jochum W, et al. Impact of integrated positron emission tomography and computed tomography on staging and management of gallbladder cancer and cholangiocarcinoma. J Hepatol. 2006 Jul;45(1):43-50.

8 Neuhaus P, Thelen A, Jonas S, Puhl G, Denecke T, Veltzke-Schlieker W, et al. Oncological superiority of hilar en bloc resection for the treatment of hilar cholangiocarcinoma. Ann Surg Oncol. 2012 May;19(5):1602-8.

9 Bednarsch J, Czigany Z, Lurje I, Tacke F, Strnad P, Ulmer TF, et al. Left- versus rightsided hepatectomy with hilar en-bloc resection in perihilar cholangiocarcinoma. HPB. 2020 Mar;22(3):437-44. 
10 Clavien PA, Petrowsky H, DeOliveira ML, Graf R. Strategies for safer liver surgery and partial liver transplantation. N Engl J Med. 2007 Apr 12;356(15):1545-59.

11 Bednarsch J, Czigany Z, Lurje I, Amygdalos I, Strnad P, Halm P, et al. Insufficient future liver remnant and preoperative cholangitis predict perioperative outcome in perihilar cholangiocarcinoma. HPB. 2021;23(1):99-108.

12 Nagino M, Ebata T, Yokoyama Y, Igami T, Sugawara G, Takahashi Y, et al. Evolution of surgical treatment for perihilar cholangiocarcinoma: a single-center 34-year review of 574 consecutive resections. Ann Surg. 2013 Jul; 258(1):129-40

13 Kimura N, Toyoki Y, Ishido K, Kudo D, Yakoshi Y, Tsutsumi S, et al. Perioperative blood transfusion as a poor prognostic factor after aggressive surgical resection for hilar cholangiocarcinoma. J Gastrointest Surg. 2015 May; 19(6):1194-5.

14 Waghray A, Sobotka A, Marrero CR, Estfan B, Aucejo F, Narayanan Menon KV. Serum albumin predicts survival in patients with hilar cholangiocarcinoma. Gastroenterol Rep. 2017 Feb;5(1):62-6.

15 Valle J, Wasan H, Palmer DH, Cunningham D, Anthoney A, Maraveyas A, et al. Cisplatin plus gemcitabine versus gemcitabine for biliary tract cancer. N Engl J Med. 2010 Apr 8; 362(14):1273-81.

16 Shirai K, Ebata T, Oda K, Nishio H, Nagasaka T, Nimura Y, et al. Perineural invasion is a prognostic factor in intrahepatic cholangiocarcinoma. World J Surg. 2008 Nov;32(11): 2395-402.

17 Fisher SB, Patel SH, Kooby DA, Weber S, Bloomston M, Cho C, et al. Lymphovascular and perineural invasion as selection criteria for adjuvant therapy in intrahepatic cholangiocarcinoma: a multi-institution analysis. HPB. 2012 Aug;14(8):514-22.

18 Zhang L, Guo L, Tao M, Fu W, Xiu D. Parasympathetic neurogenesis is strongly associated with tumor budding and correlates with an adverse prognosis in pancreatic ductal adenocarcinoma. Chin J Cancer Res. 2016 Apr; 28(2):180-6.

19 Iwasaki T, Hiraoka N, Ino Y, Nakajima K, Kishi Y, Nara S, et al. Reduction of intrapancreatic neural density in cancer tissue predicts poorer outcome in pancreatic ductal carcinoma. Cancer Sci. 2019 Apr;110(4):1491-502.

20 Bednarsch J, Czigany Z, Heise D, Lang SA, Olde Damink SWM, Luedde T, et al. Leakage and stenosis of the hepaticojejunostomy following surgery for perihilar cholangiocarcinoma. J Clin Med. 2020 May 8;9(5):1392.

21 Neuhaus P, Jonas S, Settmacher U, Thelen A, Benckert C, Lopez-Hänninen E, et al. Surgical management of proximal bile duct cancer: extended right lobe resection increases resectability and radicality. Langenbecks Arch Surg. 2003 Jul;388(3):194-200.
22 Jonas S, Benckert C, Thelen A, Lopez-Hänninen E, Rösch T, Neuhaus P. Radical surgery for hilar cholangiocarcinoma. Eur J Surg Oncol. 2008 Mar;34(3):263-71.

23 Primrose JN, Fox RP, Palmer DH, Malik HZ, Prasad R, Mirza D, et al. Capecitabine compared with observation in resected biliary tract cancer (BILCAP): a randomised, controlled, multicentre, phase 3 study. Lancet Oncol. 2019 May;20(5):663-73.

24 Banales JM, Marin JJG, Lamarca A, Rodrigues PM, Khan SA, Roberts LR, et al. Cholangiocarcinoma 2020: the next horizon in mechanisms and management. Nat Rev Gastroenterol Hepatol. 2020 Sep;17(9):557-88.

25 Chen SH, Zhang BY, Zhou B, Zhu CZ, Sun LQ, Feng YJ. Perineural invasion of cancer: a complex crosstalk between cells and molecules in the perineural niche. Am J Cancer Res. 2019;9(1):1-21.

26 Preston M, Sherman LS. Neural stem cell niches: roles for the hyaluronan-based extracellular matrix. Front Biosci. 2011 Jun 1;3: 1165-79.

27 Gritsenko PG, Ilina O, Friedl P. Interstitial guidance of cancer invasion. J Pathol. 2012 Jan;226(2):185-99.

28 Godinho-Silva C, Cardoso F, Veiga-Fernandes $H$. Neuro-immune cell units: a new paradigm in physiology. Annu Rev Immunol. 2019 Apr 26;37(1):19-46.

29 Liu HP, Tay SS, Leong S, Schemann M. Colocalization of ChAT, DbetaH and NADPH-d in the pancreatic neurons of the newborn guinea pig. Cell Tissue Res. 1998 Nov;294(2): $227-31$.

30 Franchitto A, Onori P, Renzi A, Carpino G, Mancinelli R, Alvaro D, et al. Recent advances on the mechanisms regulating cholangiocyte proliferation and the significance of the neuroendocrine regulation of cholangiocyte pathophysiology. Ann Transl Med. 2013 Oct; 1(3):27.

31 Dang N, Meng X, Song H. Nicotinic acetylcholine receptors and cancer. Biomed Rep. 2016 May;4(5):515-8.

32 Sha M, Cao J, Sun HY, Tong Y, Xia Q. Neuroendocrine regulation of cholangiocarcinoma: a status quo review. Biochim Biophys Acta Rev Cancer. 2019 Aug;1872(1):66-73.

33 Albo D, Akay CL, Marshall CL, Wilks JA, Verstovsek G, Liu H, et al. Neurogenesis in colorectal cancer is a marker of aggressive tumor behavior and poor outcomes. Cancer. 2011 Nov 1;117(21):4834-45.

34 Zhao CM, Hayakawa Y, Kodama Y, Muthupalani S, Westphalen CB, Andersen GT, et al. Denervation suppresses gastric tumorigenesis. Sci Transl Med. 2014 Aug 20;6(250): 250 ra115.

35 Jonas S, Thelen A, Benckert C, Biskup W, Neumann U, Rudolph B, et al. Extended liver resection for intrahepatic cholangiocarcinoma: a comparison of the prognostic accuracy of the fifth and sixth editions of the TNM classification. Ann Surg. 2009 Feb;249(2):303-9.
36 Ito T, Ebata T, Yokoyama Y, Igami T, Sugawara G, Mizuno T, et al. The pathologic correlation between liver and portal vein invasion in perihilar cholangiocarcinoma: evaluating the oncologic rationale for the American Joint Committee on Cancer definitions of T2 and T3 tumors. World J Surg. 2014 Dec; 38(12):3215-21.

37 Ebata T, Mizuno T, Yokoyama Y, Igami T, Sugawara G, Nagino M. Surgical resection for Bismuth type IV perihilar cholangiocarcinoma. Br J Surg. 2018;105(7):829-38.

$38 \mathrm{Hu}$ J-H, Tang J-H, Lin C-H, Chu Y-Y, Liu N-J. Preoperative staging of cholangiocarcinoma and biliary carcinoma using 18F-fluorodeoxyglucose positron emission tomography: a meta-analysis. J Investig Med. 2018 Jan;66(1): 52-61.

39 Caygill CP, Knowles RL, Hall R. Increased risk of cancer mortality after vagotomy for peptic ulcer: a preliminary analysis. Eur J Cancer Prev. 1991 Oct;1(1):35-7.

40 Partecke LI, Käding A, Trung DN, Diedrich S, Sendler M, Weiss F, et al. Subdiaphragmatic vagotomy promotes tumor growth and reduces survival via TNFa in a murine pancreatic cancer model. Oncotarget. 2017 Apr 4; 8(14):22501-12.

41 Kamiya A, Hayama Y, Kato S, Shimomura A, Shimomura T, Irie K, et al. Genetic manipulation of autonomic nerve fiber innervation and activity and its effect on breast cancer progression. Nat Neurosci. 2019 Aug;22(8): 1289-305.

42 Tibensky M, Mravec B. Role of the parasympathetic nervous system in cancer initiation and progression. Clin Transl Oncol. 2021; 23(4):669-81.

43 Heij LR, Tan X, Kather JN, Niehues JM, Sivakumar S, Heussen N, et al. Nerve fibers in the tumor microenvironment are co-localized with lymphoid aggregates in pancreatic cancer. J Clin Med. 2021;10(3):490.

44 Magnon C, Hall SJ, Lin J, Xue X, Gerber L, Freedland SJ, et al. Autonomic nerve development contributes to prostate cancer progression. Science. 2013 Jul 12;341(6142):1236361.

45 Zhang L, Wu LL, Huan HB, Chen XJ, Wen $\mathrm{XD}$, Yang DP, et al. Sympathetic and parasympathetic innervation in hepatocellular carcinoma. Neoplasma. 2017;64(6):840-6.

46 Bae GE, Kim HS, Won KY, Kim GY, Sung JY, Lim SJ. Lower sympathetic nervous system density and $\beta$-adrenoreceptor expression are involved in gastric cancer progression. Anticancer Res. 2019 Jan;39(1):231-6.

47 Zhou H, Shi B, Jia Y, Qiu G, Yang W, Li J, et al. Expression and significance of autonomic nerves and $\alpha 9$ nicotinic acetylcholine receptor in colorectal cancer. Mol Med Rep. 2018 Jun; 17(6):8423-31.

48 Hyder O, Hatzaras I, Sotiropoulos GC, Paul A, Alexandrescu S, Marques H, et al. Recurrence after operative management of intrahepatic cholangiocarcinoma. Surgery. 2013 Jun; 153(6):811-8. 
49 Komaya K, Ebata T, Yokoyama Y, Igami T, Sugawara G, Mizuno T, et al. Recurrence after curative-intent resection of perihilar cholangiocarcinoma: analysis of a large cohort with a close postoperative follow-up approach. Surgery. 2018 Apr;163(4):732-8.

50 de Jong MC, Nathan H, Sotiropoulos GC, Paul A, Alexandrescu S, Marques H, et al. Intrahepatic cholangiocarcinoma: an international multi-institutional analysis of prognostic factors and lymph node assessment. J Clin Oncol. 2011 Aug 10;29(23):3140-5.
51 Miura JT, Johnston FM, Tsai S, George B, Thomas J, Eastwood D, et al. Chemotherapy for surgically resected intrahepatic cholangiocarcinoma. Ann Surg Oncol. 2015 Oct;22(11): 3716-23.

52 Stein A, Arnold D, Bridgewater J, Goldstein D, Jensen LH, Klümpen HJ, et al. Adjuvant chemotherapy with gemcitabine and cisplatin compared to observation after curative intent resection of cholangiocarcinoma and muscle invasive gallbladder carcinoma (ACTICCA-1 trial): a randomized, multidisciplinary, multinational phase III trial. BMC Cancer. 2015;15: 564 .
53 Mizuno T, Ebata T, Yokoyama Y, Igami T, Yamaguchi J, Onoe S, et al. Combined vascular resection for locally advanced perihilar cholangiocarcinoma. Ann Surg. 2020 Sep 24.

54 Miyazaki M, Kato A, Ito H, Kimura F, Shimizu $\mathrm{H}$, Ohtsuka $\mathrm{M}$, et al. Combined vascular resection in operative resection for hilar cholangiocarcinoma: does it work or not? Surgery. 2007 May; 141(5):581-8.

55 Tamoto E, Hirano S, Tsuchikawa T, Tanaka E, Miyamoto M, Matsumoto J, et al. Portal vein resection using the no-touch technique with a hepatectomy for hilar cholangiocarcinoma. HPB. 2014 Jan;16(1):56-61. 